\title{
Stage IV Bladder Cancer AJCC v8
}

National Cancer Institute

\section{Source}

National Cancer Institute. Stage IV Bladder Cancer A/CC v8. NCI Thesaurus. Code C140424.

Stage IV includes: IVA: (T4b, N0, M0); (Any T, Any N, M1a); IVB: (Any T, Any N, M1b). T4b: Extravesical tumor invades pelvic wall, abdominal wall. N0: No regional lymph node metastasis. M0: No distant metastasis. M1a: Distant metastasis limited to lymph nodes beyond the common iliacs. M1b: Non-lymph node distant metastases. (AJCC 8th ed.) 